\title{
Host-Parasite Arms Races and Rapid Changes in Bird Egg Appearance
}

\author{
Claire N. Spottiswoode ${ }^{1,2, \star}$ and Martin Stevens ${ }^{1}$
}

1. Department of Zoology, University of Cambridge, Downing Street, Cambridge CB2 3EJ, United Kingdom; 2. Department of Science and Technology/National Research Foundation Centre of Excellence, Percy FitzPatrick Institute, University of Cape Town, Rondebosch 7701, South Africa

Submitted June 12, 2011; Accepted January 12, 2012; Electronically published March 22, 2012

Online enhancement: appendix. Dryad data: http://dx.doi.org/10.5061/dryad.ft56mn76.

\begin{abstract}
Coevolutionary arms races are a powerful force driving evolution, adaptation, and diversification. They can generate phenotypic polymorphisms that render it harder for a coevolving parasite or predator to exploit any one individual of a given species. In birds, egg polymorphisms should be an effective defense against mimetic brood parasites and are extreme in the African tawny-flanked prinia (Prinia subflava) and its parasite, the cuckoo finch (Anomalospiza imberbis). Here we use models of avian visual perception to analyze the appearance of prinia and cuckoo finch eggs from the same location over 40 years. We show that the two interacting populations have experienced rapid changes in egg traits. Egg colors of both species have diversified over time, expanding into avian color space as expected under negative frequency-dependent selection. Egg pattern showed signatures of both frequency-dependent and directional selection in different traits, which appeared to be evolving independently of one another. Host and parasite appear to be closely tracking one another's evolution, since parasites showed closer color mimicry of contemporaneous hosts. This correlational evidence suggests that hosts and parasites are locked in an ongoing arms race in egg appearance, driven by constant change in the selective advantage of different phenotypes, and that coevolutionary arms races can generate remarkably rapid phenotypic change.
\end{abstract}

Keywords: coevolution, vision, egg color, egg pattern, brood parasitism, frequency-dependent selection.

\section{Introduction}

Coevolutionary arms races between species can be powerful generators of biological diversity (Ehrlich and Raven 1964; Dawkins and Krebs 1979; Thompson 1999; Yoder and Nuismer 2010). They can drive continual evolutionary change, as host or prey species constantly alter phenotype to shake off of their parasites or predators ("Red Queen" hypothesis; Van Valen 1973; Dieckmann et al. 1995). A

\footnotetext{
* Corresponding author; e-mail: cns26@cam.ac.uk,ms726@cam.ac.uk.
}

Am. Nat. 2012. Vol. 179, pp. 633-648. (C) 2012 by The University of Chicago. 0003-0147/2012/17905-53094\$15.00. All rights reserved. DOI: $10.1086 / 665031$ key driver of such change can be apostatic (negative frequency-dependent) selection, which favors rare phenotypes in hosts or prey because common phenotypes are more susceptible to parasites or predators. In camouflaged prey, for example, rare phenotypes can be at a selective advantage because predators have not developed a search image for their appearance, thus generating polymorphism in prey appearance (Allen and Clarke 1968; Pietrewicz and Kamil 1979; Allen 1988; Bond and Kamil 2002; Bond and Kamil 2006). Similar processes may occur in host-parasite systems, including the coevolutionary interactions between avian brood parasites and their hosts, which theoretical models suggest are likely to take a form that promotes coevolutionary diversification (Yoder and Nuismer 2010). In avian brood parasites, it is well established that eggrejection behavior by host parents has repeatedly led to egg mimicry by parasites, including several species of cuckoo (Cuculus and Chrysococcyx spp.; e.g., Brooke and Davies 1988; Moksnes et al. 1990; Avilés 2008; Cassey et al. 2008; Stoddard and Stevens 2011) and the cuckoo finch (Anomalospiza imberbis; Spottiswoode and Stevens 2010). From the host perspective, laying polymorphic eggs should be an effective defense against a mimetic parasite, since diversity between clutches laid by different host females renders it less likely that a parasite can accurately match any one individual's clutch, thus facilitating egg recognition and rejection (Swynnerton 1918; Davies and Brooke 1989; Takasu 2003). Accordingly, high intraspecific variation in egg appearance has arisen independently in several bird species subject to brood parasitism and is commonly referred to as polymorphism, although the phenotypic variation is often continuous and thus does not correspond to the strict definition of classical discontinuous polymorphisms. Such polymorphisms have been shown to aid egg recognition by hosts (e.g., Victoria 1972; Lahti 2006; Spottiswoode and Stevens 2010; Yang et al. 2010), are greater in host species that have an evolutionary history 
of parasitism (e.g., Stokke et al. 2002), and diminish in the absence of parasitism (Lahti 2005). An insect analogue has recently been discovered in the ant Formica fusca, in which cuticular hydrocarbon signatures have diversified in populations exploited by several species of socially parasitic ant, permitting improved discrimination ability of egg and nest mates (Martin et al. 2011).

Red Queen dynamics might be expected to arise in such mimetic systems: any host female laying a rare egg type is more likely to evade parasitism, but as a successful rare egg type grows more common, it should in turn be tracked by mimetic parasites and ultimately suffer higher parasitism. Thus, negative frequency-dependent selection should change host and parasite phenotypes over time in a race between the host evolving new signatures and the parasite evolving new forgeries. Theoretical modeling of brood parasitic mimicry suggests that such a race can generate continuous oscillations in egg appearance (Takasu 2005), in common with broader models of antagonistic coevolutionary dynamics involving phenotype matching (e.g., Nuismer et al. 2005). However, as Takasu (2003) points out, aside from theoretical viewpoints we have "few data to estimate the temporal change of egg appearance of avian brood parasites and their hosts" (p. 356). Detecting evidence of an arms race through phenotypic space is difficult with empirical data because data sets covering a significant period of time are scarce. More broadly, studying evolutionary dynamics in nature is relatively rare because temporal data are often limited (Decaestecker et al. 2007; Yang et al. 2010).

In this study, we test for coevolutionary temporal changes in egg appearance using a sample of current-day (2007-2009) and historical (predominantly 20-30 years earlier) parasite and host eggs from the same geographical location. Our study system at a site in Zambia is the Afrotropical cuckoo finch and its hosts, which provide a very good opportunity for investigating coevolution of frequency-dependent defenses owing to the extreme levels of phenotypic polymorphism in both parties. Hosts experience strong fitness costs of parasitism (Vernon 1964) and consequently are highly adept at rejecting foreign eggs, using multiple different and independent aspects of color and pattern as cues (Spottiswoode and Stevens 2010, 2011). This has resulted in mimetic host-specific parasite races just as in common cuckoos (Cuculus canorus; Newton 1896; Brooke and Davies 1988), yet cuckoo finches face the additional challenge of mimicking multiple cooccurring color and pattern variants within host species. Their principal host is the tawny-flanked prinia (Prinia subflava; Vernon 1964), which shows perhaps the most extensive polymorphisms in egg appearance of any bird species: individual females always lay the same egg type, but among females eggs range in color from white to blue to brick red to olive green, overlaid with a wide diversity of markings (fig. 1; Spottiswoode and Stevens 2010). While cuckoo finches produce a range of corresponding phenotypes (fig. 1), individual parasites do not target specific egg colors within the host population and instead rely on occasional chance matches in egg appearance to succeed (Spottiswoode and Stevens 2010). They thus incur high degrees of loss through host rejection, which underscores the efficacy of egg polymorphisms as a host defense. Previously, we have shown that prinia hosts use several aspects of egg appearance to reject foreign eggs, and these correspond to those features of egg appearance that convey the most reliable information about egg identity, since they are precisely the traits that differ most between real parasitic and host eggs (Spottiswoode and Stevens 2010). Thus, multiple aspects of egg appearance appear to be under selection from host-parasite interactions in this system.

Our first prediction is that phenotypic diversity in egg appearance should have changed over time in both parties, as host phenotypes diversify or contract under negative frequency-dependent selection and parasitic phenotypes follow. Different aspects of egg appearance may oscillate over time in phenotypic space; although some traits may be expanding (increasing) in diversity, physiological limits and other selection pressures will prevent a continuous

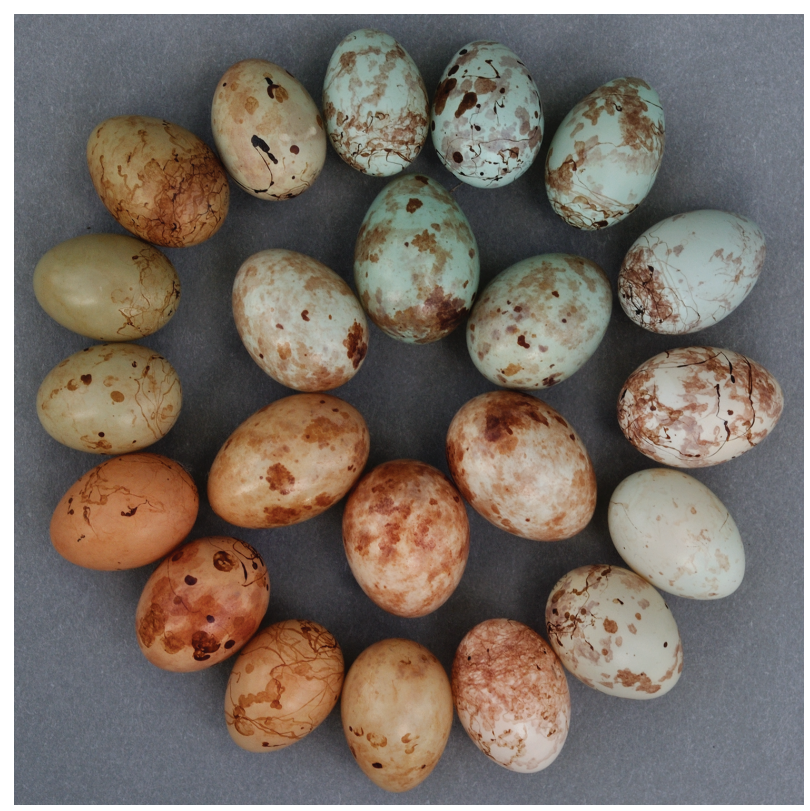

Figure 1: Eggs of tawny-flanked prinias (outer ring) and cuckoo finches (inner ring), arranged to mimic their respective distributions in avian color space. Egg colors vary continuously (clockwise from top left) from olive through blue and white to red. 
expansion, and we might expect some traits to be contracting in diversity when parasitic eggs at the center of the distribution become rare and host phenotypes at the center of the distribution therefore become newly advantageous. Different traits may be at different stages in such a cycle, and the key prediction is that we expect to find changes over time in visual attributes of eggs.

Under a scenario of fluctuating selection, in which parasites closely track hosts over time, parasitic infectivity is expected to be greatest between contemporaneous hosts and parasites compared with hosts and parasites from different time periods (Gandon et al. 2008). A recent study of Daphnia and its microparasites was able to show precisely such a pattern by reviving dormant stages of both species from a temporal series of lake sediments and comparing infectivity within and across time periods (Decaestecker et al. 2007). In our system, historical hosts and parasites cannot be revived, but "infectivity" is closely reflected by the degree of phenotypic matching (mimicry) between parasite and host, since this strongly influences a parasitic egg's probability of acceptance by the host parents (Spottiswoode and Stevens 2010). Hence, our second prediction is that if parasite evolution is closely tracking host evolution, then parasitic eggs should be a better phenotypic match to host eggs from the same time period than to host eggs from a different period. We further predict that the latter effect should be most pronounced when comparing historical host eggs to current-day parasites that hosts have not yet encountered in their evolutionary history; in contrast, a smaller effect might be expected when comparing current-day hosts to historical parasites, which have previously imposed selection on the host population.

Finally, we predict that parasites should show less phenotypic diversity than hosts, owing to a time lag between host and parasite adaptation. To test these predictions while taking bird vision into account, we calculated perceptual measures of egg color using models of avian vision and, using digital image analysis, quantified several aspects of egg pattern (dispersion, contrast, marking size, pattern coverage, and pattern diversity) that have been shown to play a role in egg rejection in this system (Spottiswoode and Stevens 2010) and to be mimicked by brood parasites in another system (Stoddard and Stevens 2010).

In summary, in this study we investigate whether changes have occurred in the appearance of host and parasitic eggs over time, as predicted by coevolutionary interactions. We use models of avian vision and digital image analysis to quantify the nature and degree of change with respect to color diversity, principal color types, and various aspects of pattern. Finally, we relate our findings to potential models that may explain coevolutionary dynamics in this system.

\section{Material and Methods \\ Fieldwork and Data Sets}

Cuckoo finches and prinias were studied in the Choma District of southern Zambia within an $8-\mathrm{km}$ radius of $16^{\circ} 47^{\prime} \mathrm{S}, 26^{\circ} 50^{\prime} \mathrm{E}$, but mainly on Musumanene Farm $\left(16^{\circ} 47^{\prime} \mathrm{S}, 26^{\circ} 54^{\prime} \mathrm{E}\right)$ and adjoining farms. The habitat is a mosaic of overgrown agricultural fields, natural grassland, and miombo woodland, where prinias are abundant and at least $19 \%$ of nests experience parasitism attempts. The geographical area of the current-day sampling was a subset within that of the historical sampling, rendering our findings conservative. Current-day fieldwork was carried out during January-March of $2007-2009$ by C.N.S., and the historical collection was gathered from 1969 to 2002 (91\% of clutches were collected during the 1970s and 1980s) by J. F. R. Colebrook-Robjent. The latter forms part of a private egg collection bequeathed to the Natural History Museum, Tring, United Kingdom. Current-day eggs were randomly drawn from the population and were either single eggs removed from active nests as part of simultaneous egg-rejection experiments (Spottiswoode and Stevens 2010) or deserted clutches (single deserted eggs commonly result from mistimed parasitism attempts). If more than one egg per clutch was available, one was randomly selected for analysis, to avoid pseudoreplication. Repeat layings within years were excluded.

\section{Modeling in Bird Color Space}

We first determined the phenotypic resemblance among and between parasitic and host eggs in terms of avian vision by analyzing both the background coloration and the pattern of overlaid darker markings, such as spots, blotches, and fine lines (fig. 1). To quantify color, we carried out spectrophotometry to obtain reflectance spectra of eggs, indoors and under constant light conditions. Measurements were taken using an USB2000 spectrophotometer, with a PX-2 pulsed xenon light source and an R4007-UV/VIS reflectance probe (all Ocean Optics, Dunedin, FL), with reference to a Spectralon $99 \%$ white reflectance standard (Labsphere, Congleton, United Kingdom). Each egg was held at a constant distance $(5 \mathrm{~mm})$ and angle $\left(45^{\circ}\right)$ from the probe tip by means of a slanted plastic sleeve attached to the probe. Five measurements were taken of the egg's background color (i.e., avoiding overlaid darker markings) throughout the egg, and the mean was analyzed. Irradiance (ambient light) within nests was measured in the field (during sunny conditions between 1100 and 1400 hours) using a cosine-corrected probe (Ocean Optics) attached to the spectrophotometer, to obtain readings of irradiance over a wide angle. Five measurements 
were taken at different angles within each of five nests, and the mean was analyzed.

It is essential to analyze egg appearance in terms of bird vision because birds have a different and more complex color vision system than do humans. Birds have four single-cone types (ultraviolet [UV], shortwave [SW], mediumwave $[\mathrm{MW}]$, and longwave $[\mathrm{LW}]$ ) responsible for color vision (Cuthill 2006). We used reflectance and irradiance spectra to calculate the predicted photon catches of each single cone, following Endler and Mielke (2005). This approach requires spectral sensitivity data for the bird's retina, which are unavailable for the host species in our study; therefore, we used visual sensitivities of the blue tit (Cyanistes caeruleus; Hart et al. 2000b) and the zebra finch (Taeniopygia guttata; Hart et al. 2000a) as model species. Double-cone sensitivity data, used to calculate luminance ("lightness") information (Osorio and Vorobyev 2005), were available only for the blue tit. These model species are distantly related, are geographically separated, and occupy different light environments, so they offer a good test to determine the level of error that may be associated with not knowing the sensitivity of the host species in this study. There was no difference between the two visual systems in terms of the predicted photon catch values (less than $0.05 \pm 0.01 \mathrm{SD}$ average difference between the blue tit and the zebra finch values for each single-cone type), as expected since visual sensitivities among higher passerines seem to be strongly conserved (Ödeen and Håstad 2003; Cuthill 2006). Therefore, we present the analysis of egg colors only with respect to the better-studied blue tit visual system. Experimental evidence from the prinia-cuckoo finch system indicates that host rejection behavior depends principally on color and pattern differences and that differences in luminance are not an important cue (Spottiswoode and Stevens 2010), as expected since discrimination tasks under moderate to high light levels seem primarily to involve color vision (Vorobyev and Osorio 1998; Osorio and Vorobyev 2005). Therefore, we analyzed changes in egg appearance for color but not luminance. We used analysis of luminance to check for changes in egg appearance produced by removing the contents of the eggs (see below).

\section{Calculating Perceptual Differences in Color}

The cone catches described above provide a measure of the photoreceptor stimulation of individual eggs in avian vision. Using these, we calculated a measure of perceptual distance between pairs of eggs, to estimate phenotypic diversity in perceptual units relevant to visual discrimination by hosts (see "Quantifying Phenotypic Diversity in Egg Traits" below). To do so, we used a log form of a discriminability model of avian visual processing, which assumes that receptor noise limits visual discrimination (Vorobyev and Osorio 1998), using single-cone proportions for the blue tit $(\mathrm{LW}=1.00, \mathrm{MW}=0.99, \mathrm{SW}=0.71$, and $\mathrm{UV}$ sensitive $=0.37$; Hart et al. 2000b) and a Weber fraction of 0.05. Color vision in birds stems from the four singlecone types (Cuthill 2006), and we therefore used the tetrachromatic version of the model with the single cones to model color discrimination (Vorobyev and Osorio 1998). The output of the model is in "just noticeable differences" (jnds), where each jnd value corresponds to a single unit of color discrimination (Siddiqi et al. 2004).

\section{Quantifying Eggshell Pattern}

Egg appearance (and rejection by hosts) is also influenced by the pattern of overlaid darker markings (e.g., spots, blotches, and fine lines; fig. 1) as well as by color. We therefore quantified eggshell patterns by following our previous approach that used calibrated digital images (Stevens et al. 2007) to derive five uncorrelated measures of pattern (full description in Spottiswoode and Stevens 2010; Stoddard and Stevens 2010). Briefly, we used a "granularity" analysis (Hanlon et al. 2009) involving Fourier transformation and bandpass filtering to decompose the original images of the eggs into seven new images, each capturing information at different spatial frequencies. We calculated the energy in each image as the sum of the squared pixel values divided by the image size (Hanlon et al. 2009; Stoddard and Stevens 2010), which tells us how much information at each spatial scale is present. The values across all seven images produces a granularity spectrum. From this spectrum we calculated the image (spatial scale) with the highest energy, which reflects the marking size that is most prevalent in the overall egg pattern ("filter size," which is an inverse measure of marking size; i.e., smaller filter sizes capture information about larger markings). The proportion of the energy that the filter with the dominant marking size contributes to the total energy across all scales is a measure of how much this marking size dominates ("proportion energy"). A large value indicates that an egg's patterning is dominated by just one marking size. The total energy, or amplitude, of the entire granularity spectrum is a measure of how contrasting the markings are overall ("total energy"). Finally, we obtained two further measurements of pattern based on Stoddard and Stevens (2010). We thresholded egg images into a binary format, with 0 encoding egg background color and 1 encoding pattern. From this we calculated the average area of the egg that was covered with patterning ("proportion coverage") and the difference in pattern between the narrow and broad ends of the egg ("dispersion"). To quantify egg-pattern diversity, we used the same approach described 
above for color. Analyses were undertaken with customwritten programs in MATLAB (Mathworks).

It should be noted that although our measurements of pattern are broadly inspired by the way that vertebrate spatial vision works (i.e., decomposition of a visual scene into different spatial frequencies), we do not know exactly what levels of pattern trait difference are perceptually salient. Therefore, unlike for color we cannot relate our measurements of pattern to units of visual discrimination based on formal physiological models. However, we note that in egg-rejection experiments three of the five pattern traits have been experimentally shown to predict rejection behavior in this host species (and a fourth pattern attribute in another host species; Spottiswoode and Stevens 2010, 2011). This suggests that the pattern traits we have analyzed are biologically meaningful with respect to selection on egg patterning.

\section{Quantifying Phenotypic Diversity in Egg Traits}

To test for changes in degree of polymorphism over time, we required a measure of phenotypic diversity for each color or pattern trait. We generated such a measure by calculating the phenotypic distance for that trait between each egg and every other egg in the sample, producing a matrix of distances. These were perceptual distances (jnds; see above) in the case of color and absolute distances in the case of pattern. Phenotypic diversity for each trait was defined as the grand mean of the mean distances between each egg and every other egg in the population. High values indicate that eggs are on average farther apart from one another in phenotypic space and hence show greater phenotypic diversity. We also quantified the type of color change that may have occurred, including changes in the principal egg colors and the color extremity of the eggs with respect to the center of the distribution (an egg of average color; see "Results").

\section{Comparing Blown versus Unblown Eggs}

Finally, to compare the historical and current-day samples we needed to check that their condition did not affect their appearance. This is because the historical eggs were empty shells (blown) in a museum collection, whereas the current-day eggs were freshly collected and still had their contents intact (unblown). We assessed any potential change in egg appearance for color and luminance as a consequence of blowing by comparing the values of a set of the same eggs $(N=34$, host and parasite) measured when unblown and again when blown. We modeled the photon catches for each egg before and after blowing and compared these paired values for each cone type with Wilcoxon signed-rank matched-pairs tests. There were no sig- nificant differences between the photon catch values of the blown versus unblown eggs for any of the cone types (fig. A1A, available online; UV: $V=318, P=.74$; SW: $V=$ 252, $P=.45$; MW: $V=303, P=.93$; LW: $V=370$, $P=.22$; double: $V=326, P=.64)$.

To confirm that there was no perceptual change in egg appearance, we also used the avian visual discrimination model described above (see "Calculating Perceptual Differences in Color"). In addition to color perception, which stems from the single cones, luminance-based tasks apparently stem from the double cones in birds (Osorio and Vorobyev 2005). Therefore, we used the tetrachromatic version of the model to analyze color discrimination (Vorobyev and Osorio 1998) and the double cones for luminance discrimination (Siddiqi et al. 2004). The output of the model is in jnds (see above). Generally, a jnd value of less than 3 indicates that two stimuli are only likely to be distinguishable under optimal light levels (Siddiqi et al. 2004), which is not the case in our system. The results showed that it is highly unlikely that there is a perceptible change in appearance from the unblown to blown eggs, as the jnd values were very low for both color (mean jnd $=1.53, \mathrm{SD}=1.18$ ) and luminance (mean jnd $=$ $1.45, \mathrm{SD}=1.51$; fig. $\mathrm{A} 1 B$ ). We can therefore be confident in comparing blown and unblown eggs with respect to both color and luminance.

\section{Statistical Analyses}

We tested for spatial patterns in egg appearance using Mantel tests (with 999 replicates) on matrices of geographical distances calculated from GPS coordinates of nests and Euclidean distances among $x, y$, and $z$ coordinates of eggs in avian color space, as well as each of the five pattern traits. Analyses of changes in jnd and pattern distances used unequal-variance $t$-tests on ranked data (as recommended by Ruxton [2006] owing to departures from normality and homogeneity of variances), implemented in $\mathrm{R}$ (R Development Core Team 2010). For all tests involving trait diversity, we conducted resampling analyses $(N=$ 999 resamples) to correct for differences in sample size between species and time periods; in each case, the number of eggs resampled was equal to the sample size for the smallest group in the analysis. Analyses of color "angle" based on opponency-style calculations of single-cone values used circular statistics (see below), implemented by the R package circular (Lund and Agostinelli 2007). Our data did not conform to a von Mises distribution, which parametric circular statistics usually require, so we used nonparametric Watson two-sample tests. 


\section{Results}

Distribution of Eggs in Geographical and Phenotypic Space

First, we tested whether there was any geographical spatial pattern in egg appearance of current-day eggs and found none, either in color $(N=301, P=.4)$ or in any aspect of pattern $(N=243, P>.3)$. This confirmed that egg color and pattern variants co-occurred and that spatial information is highly unlikely to provide parasites with a potential cue to host egg phenotype, corroborating the frequent incidence of parasitic eggs in host clutches that strongly differed from them in appearance (Spottiswoode and Stevens 2010).

Second, we carried out an initial investigation of the distribution of host and parasite egg colors in tetrahedral avian color space by standardizing single-cone catch values for each egg (thus removing differences in absolute brightness) and converting them into coordinates in avian color space (Endler and Mielke 2005). Initial inspection of such plots for the current-day data set indicated that all the variation in egg color in both species lay along one main plane in color space (fig. A2, available online; $n=362$ eggs). This was confirmed by a principal component (PC) analysis on a covariance matrix of the standardized four cone catch values: two PCs explained $99.5 \%$ of all variation in egg appearance (PC1 corresponds to $72.0 \%$ of the variation). PC1 coefficients were as follows: UV: 0.116; SW: 0.600; MW: 0.072; and LW: -0.788 . For PC2, the coefficients were as follows: UV: -0.836 ; SW: 0.250 ; MW: 0.475; and LW: 0.111. These PCs provided a basis for encoding color information in a biologically relevant way, with two color channels broadly corresponding to potential opponent color channels in the visual system (Komdeur et al. 2005). The two color channels (CCs) can be expressed as (Spottiswoode and Stevens 2011)

$$
\mathrm{CC} 1=\frac{\mathrm{LW}}{(\mathrm{UV}+\mathrm{SW}+\mathrm{MW}) / 3}
$$

and

$$
\mathrm{CC} 2=\frac{(\mathrm{SW}+\mathrm{MW}+\mathrm{LW}) / 3}{\mathrm{UV}}
$$

where UV, SW, MW, and LW respectively refer to the ultraviolet and short-, medium-, and longwave standardized cone catches. These color channels can be used as Cartesian coordinates to plot egg colors in two dimensions and to carry out circular statistics (see below), while our analyses of color diversity remain based on color perceptual distances.

A striking feature of the planar egg color distribution was a central "hole" in which no eggs fell, resulting in a doughnutlike distribution in bird color space (figs. A2, A3, available online). On inspection it appeared to coincide closely with the center of the distribution of egg colors. To confirm this, we (i) calculated the center of the color distribution by taking the geometric mean of the two color channels (separately for the historical and current data sets, with their arithmetic mean taken) and (ii) calculated the center of the hole by manually selecting those eggs distributed around the edges of the hole and then calculating the point within the hole that minimized the total Euclidian distance to all the surrounding egg values. Figure A4, available online, shows that these two points coincided closely, which implies that average-colored eggs are absent from both historical and current-day samples. Neither point was close to the achromatic ("gray") point in bird color space, which is biologically reasonable since the population average should be constrained by available pigments. We can speculate that the existence of the hole is simply a by-product of eggs moving apart in color space; as the eggs spread outward, a gap is left in the center of the distribution. Alternatively, it may also exist if both host and parasite "average-colored" eggs are disadvantageous because such host eggs may be susceptible to parasites from all sides of the color distribution, and parasite eggs would not match any host eggs (see "Discussion").

\section{Prediction 1: Eggs Have Changed Their Distribution in Phenotypic Space over Time}

Color. To test for temporal change, we first used our index of phenotypic diversity expressed in discrimination units ("Material and Methods"). We found that host eggs have moved significantly farther apart from one another in perceptual color space (fig. $2 ; t_{230.38}^{\prime}=13.51, P<.001$ for full data set; $P<.001$ for $100 \%$ of resamples with $N=128$ ), as have parasitic eggs $\left(t_{61.96}^{\prime}=3.62, P<.001\right.$ for full data set; $P<.05$ for $56.8 \%$ of resamples with $N=30$ ). This indicates that the amount of avian color space occupied by the current-day sample was larger than that occupied by the historical sample; therefore, prediction 1 was clearly supported.

We also tested for temporal change using a second measure. Overall diversity of colors can also be estimated by the total volume occupied in tetrahedral avian color space, defined by a minimum convex polygon containing all points corresponding to the distribution of egg colors (Stoddard and Prum 2008). This approach showed a marked expansion in total volume occupied both by hosts and by parasites, increasing over time by factors of 5.40 and 7.26, respectively (fig. A5, available online); resampling confirmed that about a threefold change occurred even when controlling for sample size (mean volume of resampled current-day eggs was 3.82 [SD $=0.49$ ] times that of the historical eggs for hosts and 3.90 [SD $=1.21$ ] 

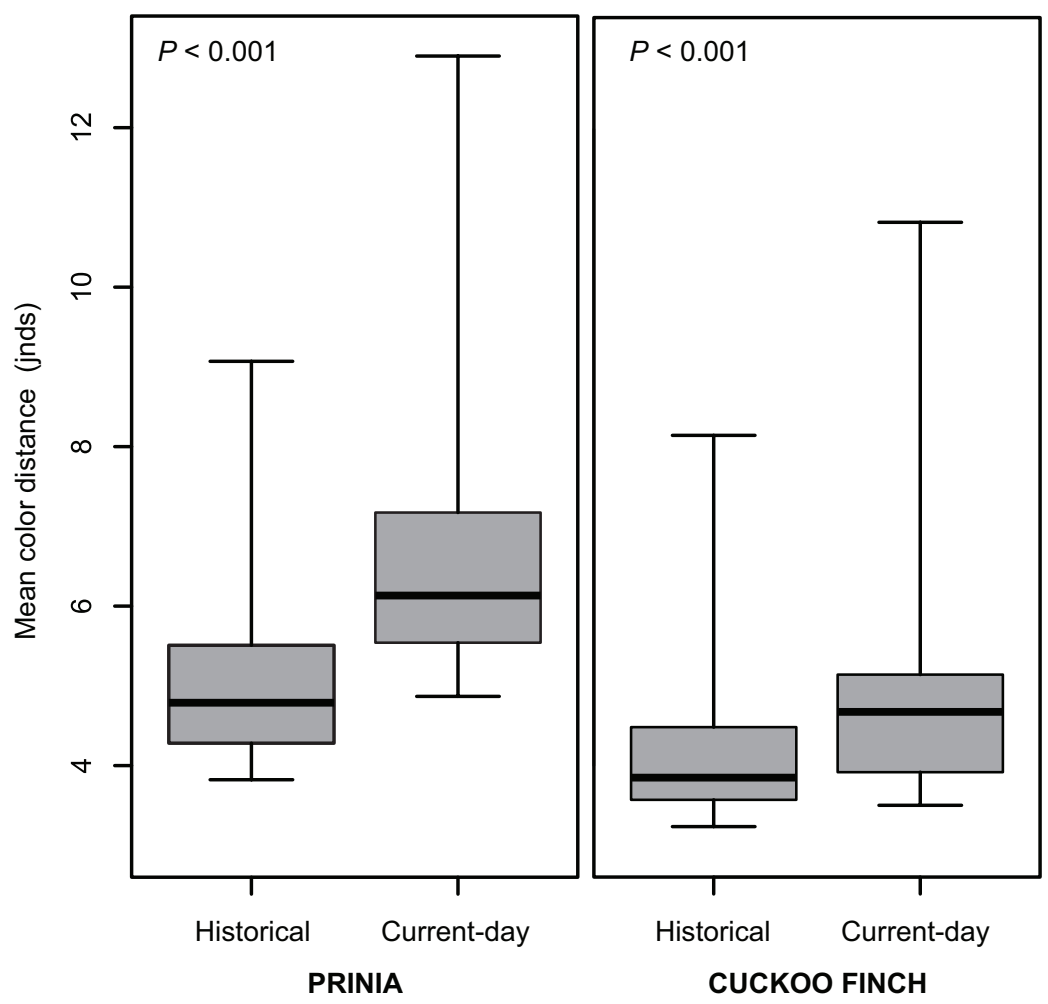

Figure 2: Perceptual color distances (in "just noticeable differences" [jnds]) between eggs in the historical and current-day data sets for both the host and cuckoo finch, showing that in both parties current-day eggs were significantly more spread out from each other (and hence more diverse in coloration) than were historical eggs. Whiskers show ranges.

times for parasites). Thus, both approaches revealed an increase in egg color diversity over time.

The analyses described above show that egg colors have become more diverse. However, they do not show exactly how such changes have been brought about: discrimination thresholds (jnds) only reveal the magnitude of color change, which could occur in any direction and be of any type. Two non-mutually-exclusive mechanisms might contribute to changes in color diversity: changes in color type ("hue") and changes in color extremity (distance from the average color of the distribution). A third analytical approach allowing these two mechanisms to be distinguished was suggested by the planar distribution of egg colors in tetrahedral color space ("Distribution of Eggs in Geographical and Phenotypic Space"), allowing us to express egg colors in Cartesian coordinates defined by color channels. First, we encoded the position of every egg in relation to the center of the color distribution based on the color channel values, which describes deviations from an average phenotype. We then shifted the distribution such that the origin lay at its center (based on the geometric mean of the egg colors; "Distribution of Eggs in Geographical and Phenotypic Space"). Then we converted Cartesian coor- dinates for each egg into polar coordinates. Polar coordinates encode the egg's distance from the center of the distribution (reflecting its color extremity), and its angle in degrees around the hole (reflecting its color type, or hue).

Using circular statistics ("Material and Methods"), we found that egg color frequency (hue) had changed over time: there were significant changes in the median color angle between the historical and current-day egg collections, both for prinias (Watson's two-sample test statistic $=0.762, \quad P<.001 ; \quad N \quad$ historical $=128, \quad N$ current $=304$; historical median angle $=79.2^{\circ}$, current median angle $=305.3^{\circ}$; fig. $3 \mathrm{~A}$ ) and for cuckoo finches (test statistic $=1.047, P<.001 ; N$ historical $=30, N$ current $=58$; historical median angle $=93.6^{\circ}$, current median angle $=298.8^{\circ}$; fig. $3 B$ ). Resampling analysis again showed that this result was robust despite differences in sample size $(P<.01$ for $100 \%$ of both prinia and cuckoo finch resamples). In addition, prinia eggs had also changed over time with respect to the extremity of eggs colors: current-day prinia eggs were significantly farther away from the center of the color distribution than were historical eggs (unequal-variance $t$-test on 


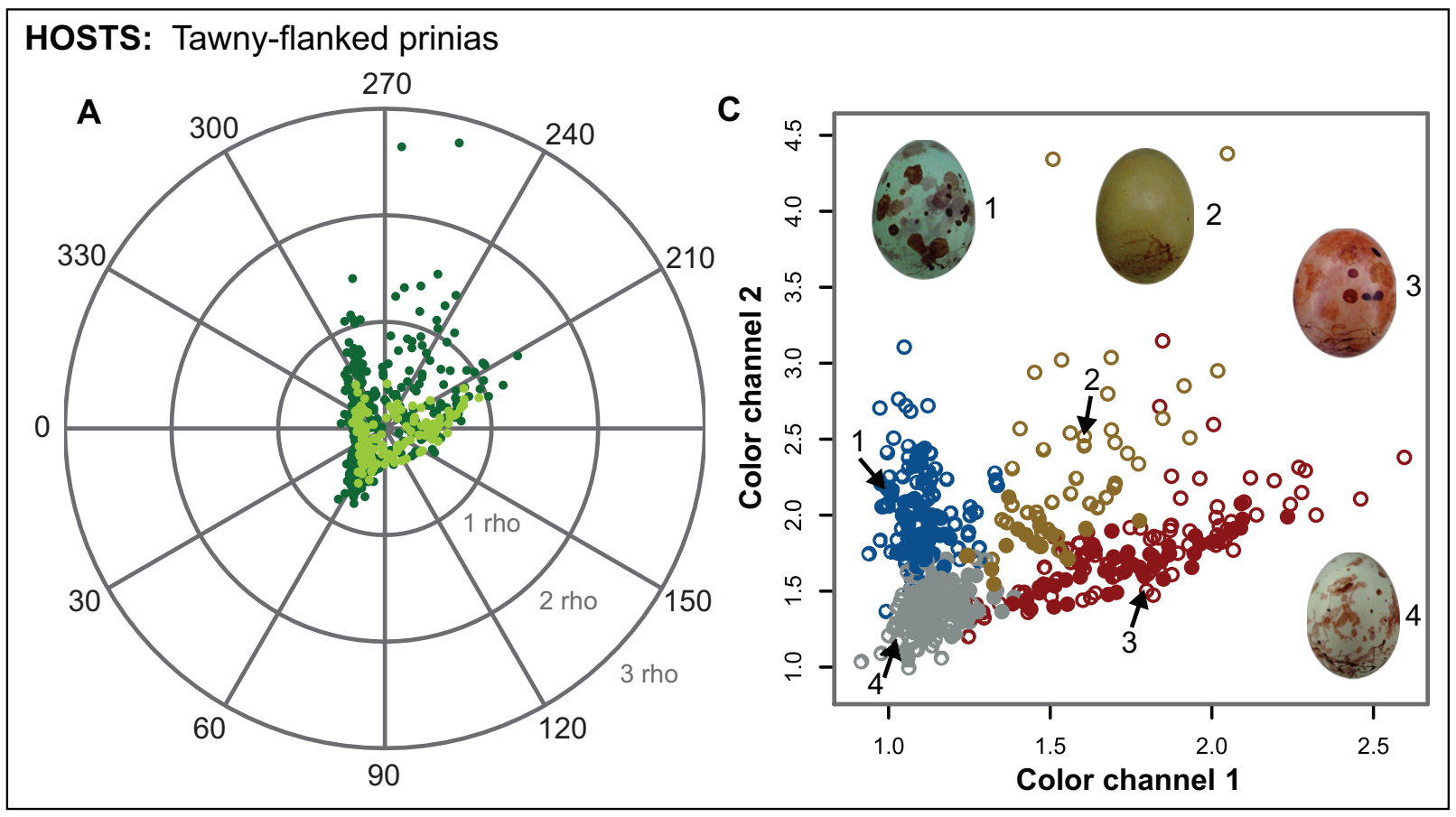

PARASITES: Cuckoo finches
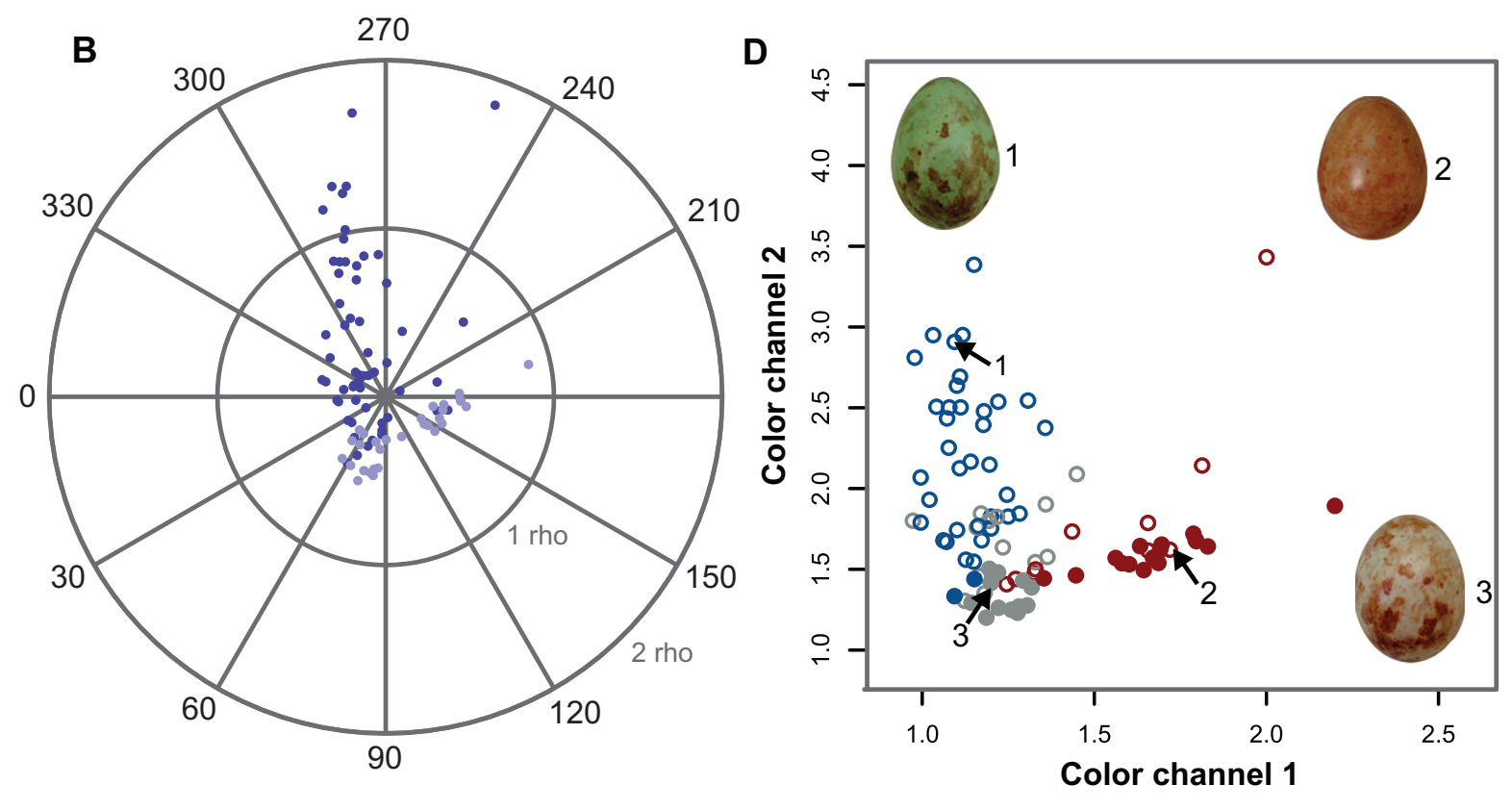

Figure 3: Changes in egg color through time. $A$ and $B$ are polar plots showing positions in color space of current-day (dark symbols) and historical (light symbols) eggs for host and parasite. Circular statistics showed that changes in median color angle through time were highly significant for both prinias $(A)$ and cuckoo finches $(B)$. Results were highly robust to differences in sample sizes between time periods. $C$ and $D$ show prinia and cuckoo finch eggs plotted according to two color channels (corresponding to their position on the plane in fig. A1, available online) and colored in broad categories by their appearance to human eyes (positions of example eggs are indicated by arrows). Filled circles show historical eggs, and open circles show current-day eggs. Over time, prinia eggs have become more extreme in color, and olive-colored eggs have especially proliferated. Cuckoo finch eggs have most strikingly switched from red to blue eggs being predominant in the sample. 
ranked data: $t_{270.60}^{\prime}=8.32, P<.001$; mean distances $\pm \mathrm{SE}$ : historical $=0.346 \pm 0.014 \quad$ rho, $\quad$ current $=0.534 \pm$ 0.018 rho). A corresponding trend for cuckoo finches was not statistically significant $\left(t_{85.57}^{\prime}=-0.02, P=.98\right.$; mean distances \pm SE: historical $=0.383 \pm 0.023$, current $=$ $0.515 \pm 0.053)$. When we repeated these analyses using the center of the hole rather than the center of the color distribution (see above), results were extremely similar; the only qualitative difference was that the increase in distance for cuckoo finches over time was statistically significant in magnitude $\left(t_{79.25}^{\prime}=2.01, P=.047\right)$.

Pattern. Among the five egg-pattern variables, some traits had become more spread out in phenotypic space, whereas others had contracted (table 1). This was the case for both host and parasite, and changes were in the same direction for both parties except for marking size ("filter size"), which became less variable in hosts but more variable in parasites. Unlike changes in trait variability, changes in mean phenotypic values indicate directional shifts in phenotype (for example, a change from large to small markings versus greater diversity of marking sizes among individuals). Mean phenotypic trait values changed for some traits but remained unchanged for others (table 1). Lack of change in mean trait values was generally associated with increased variability, whereas significant changes in mean trait values were generally associated with decreased variability (table 1). For example, the proportion of the egg covered by markings (pattern proportion) did not change on average over time, but eggs are currently significantly more variable with respect to proportion coverage than they were historically. Conversely, an inverse measure of marking size (filter size) has over time become less variable among clutches, but egg patterns are on average larger than they were in the past. The only exception was pattern dispersion in parasites: over time egg patterns have become more evenly distributed across the egg and simultaneously more variable among nests. In summary, pattern traits changed in a more complex manner than color, with some traits showing signals of diversifying selection (increases in variance but no change in mean values), whereas others showed signals of directional selection (decreases in variance and directional change in mean values; Endler 1986). As discussed above ("Material and Methods"), further modeling of pattern vision needs to be developed to understand the level of changes in terms of perceptual rather than objective differences (as is currently possible with color).

\section{Prediction 2: Hosts Should Be Better Matched by Parasites from the Same Time Period}

We compared host-parasite matching between and within time periods for both color and pattern traits. In color perceptual space, historical hosts were significantly better matched (i.e., closer in perceptual space) by historical parasites than by current-day parasites (fig. $4 ; t_{253.15}^{\prime}=$ $-4.313, P<.001$ for full data set; $P<.05$ for $94.0 \%$ of resamples with $N=30$ parasites). Current-day hosts were also better matched by current-day parasites than by historical parasites, but this was not as pronounced $\left(t_{604.99}^{\prime}=1.99, P=.048\right.$ for full data set; $P<.05$ for $26.1 \%$ of resamples with $N=128$ hosts and $N=30$ parasites). Considering only hosts and parasites from the same time period, host-parasite matching was closer in the historical sample than in the current-day sample $\left(t_{251.67}^{\prime}=6.122, P<.001 ; P<.05\right.$ for $98.8 \%$ of resamples with $N=128$ hosts and $N=30$ parasites). These results suggest that parasite evolution is closely tracking host evolution and that hosts might be diversifying faster than parasites.

The situation is more complex for pattern (table A1, available online). Pattern dispersion and contrast in current-day parasites better mimic current-day hosts than past hosts, whereas in historical parasites these traits showed poorer mimicry of contemporaneous hosts versus hosts that parasites had not yet encountered. This suggests that for these traits the parasite is evolving faster than the host, thus improving in mimicry. By contrast, for marking size and proportion coverage current-day parasites are poorer mimics of current-day hosts than they are of historical hosts, while historical parasites are better mimics of contemporaneous hosts versus hosts that they had not yet encountered. This suggests that with respect to these traits the parasite is evolving more slowly than the host and thus growing poorer in mimicry. Overall, these results indicate that pattern traits appear to be evolving independently of one another.

\section{Prediction 3: Hosts Should Be More Variable than Parasites}

Hosts were significantly more variable in egg color (in perceptual color space expressed in discrimination units) than were parasites, both within the current-day data set $\left(t_{78.00}^{\prime}=-9.52, P<.001\right.$ for full data set; $P<.05$ for $99.9 \%$ of resamples with $N=58$ ) and within the historical one $\left(t_{42.88}^{\prime}=-5.57, P<.001\right.$ for full data set; $P<.05$ for $83.5 \%$ of resamples with $N=30$ ). The volume in avian color space occupied by host eggs was also 3.62 times greater for the current-day data set and 4.86 times greater for the historical data set than that occupied by parasite eggs (fig. A5), again robust to resampling: for the current-day sample, volume for the resampled host eggs was 1.76 $(\mathrm{SD}=0.33)$ times that of parasitic eggs; for the historical sample, resampled host eggs occupied a volume 2.45 $(\mathrm{SD}=0.44)$ times that of parasitic eggs. In terms of polar 


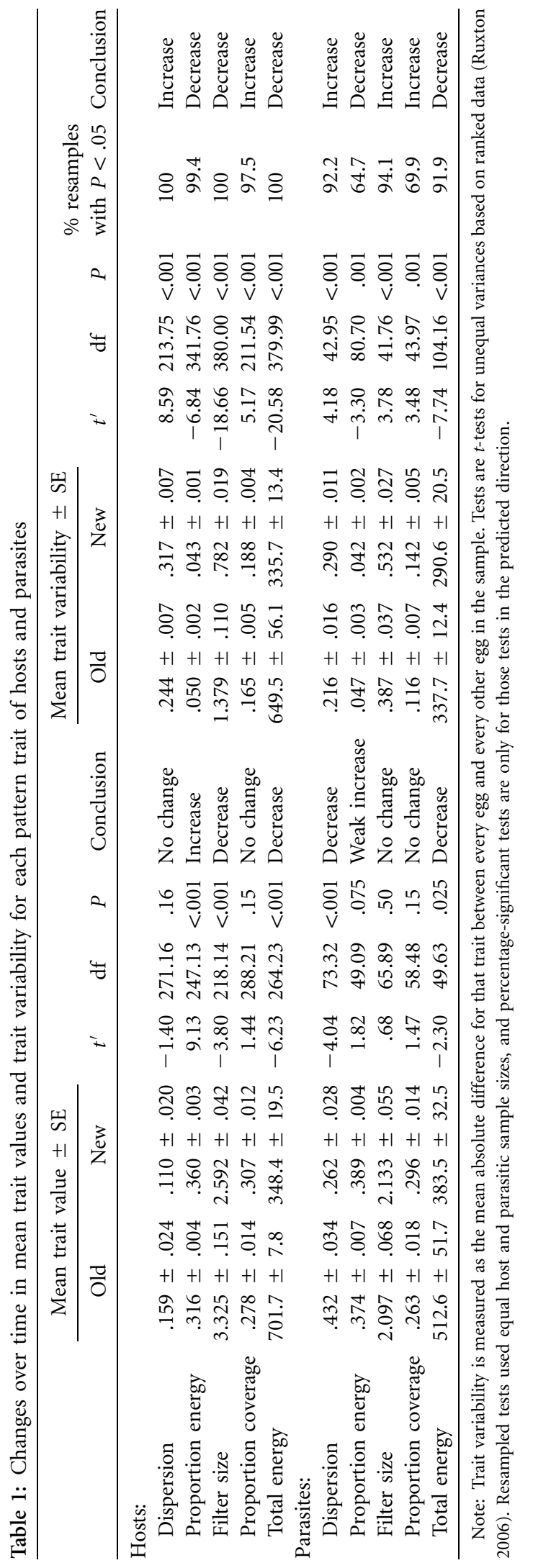






Figure 4: Time-shift analyses comparing the phenotypic distance in color between contemporaneous and noncontemporaneous hosts and parasites. Parasites were better color mimics (i.e., were closer in phenotypic space) of contemporaneous hosts than of noncontemporaneous hosts, and this difference was most pronounced for hosts that parasites had not yet encountered (see "Results").

coordinates, phenotypic distances from the center of the color distribution were substantially greater in range for prinias (historical: 0.064-0.923; current day: 0.032-2.768) than for cuckoo finches (historical: 0.248-0.870; current day: 0.091-1.850), although the mean was greater for cuckoo finches in the historical $\left(t_{55.28}^{\prime}=2.56, P=.013\right)$ but not the current-day $\left(t_{70.72}^{\prime}=-1.68, P=.098\right)$ data sets. When the center of the hole was instead used as the origin, ranges were similar to those given above, and means did not differ between species (historical: $t_{45.67}^{\prime}=0.58$, $P=.56$; current day: $\left.t_{70.84}^{\prime}=-1.29, P=.20\right)$.

In terms of eggshell patterning, hosts were more variable than parasites in all traits, significantly so for all but proportion energy; this was consistent within both the current-day and the historical data sets and was robust to resampling (table A2, available online). Prediction 3 was therefore clearly supported with respect to both color and pattern.

\section{Discussion}

This study shows that egg phenotypes in a coevolved hostparasite system have changed markedly within just a few decades. In this system, egg color has previously been shown to be the single most important cue for host rejection of foreign eggs and thus under strong selection (Spottiswoode and Stevens 2010). The present study shows that in both host and parasite there has been a significant increase in variation in egg color over time. Egg colors fell on a plane in avian color space, which might result from the very small number of pigments involved in the coloration of eggshells (Kennedy and Vevers 1976; Gorchein et al. 2009). This planar distribution allowed complementary analyses using circular statistics, which suggested that the increase in egg color variation results from both a shift in predominant egg colors in both parties and an expansion toward more diverse and extreme colors in hosts.

Such an expansion is expected under a scenario of negative frequency-dependent selection, whereby host egg colors have diversified in order to evade susceptibility to their pursuing parasite (Yoder and Nuismer 2010). Thus, there is a close parallel with other products of antagonistic coevolution, such as major histocompatibility complex allele diversity (reviewed by Apanius et al. 1997; Summers et al. 2003) and polymorphic appearance in camouflaged prey 
(e.g., Bond and Kamil 1998; Olendorf et al. 2006), in which high degrees of polymorphism are currently observed. Correspondingly, a reduction in phenotypic diversity has been detected in birds' eggs released from selection by brood parasites on a similarly short timescale (albeit centuries rather than decades; Lahti 2005).

Given that several pattern traits have also been experimentally shown to be currently under selection from discriminating hosts in this system (Spottiswoode and Stevens 2010), we might expect temporal change in eggshell patterning as well as color. A similar increase in variation was also found for certain pattern traits, while other pattern traits showed decreases in phenotypic diversity, accompanied by changes in their mean trait values. This is suggestive of directional rather than diversifying selection (Endler 1986). These results suggest that different color and pattern traits are evolving independently of one another and may currently be at different stages of coevolutionary interaction. Such an interpretation is further supported by the observation that the various color and pattern traits have very low levels of correlation and therefore have the potential to convey greater information about egg identity (Spottiswoode and Stevens 2010, 2011). Such an independent response to selection of different egg color and pattern traits would be adaptive if it increased the information content of host egg appearance (Beecher 1982; Dale et al. 2001) and thus a host's probability of detecting a parasitic egg.

These conclusions were supported by analyses comparing host-parasite matching between time periods ("timeshift analyses"; Decaestecker et al. 2007; Gaba and Ebert 2009): theoretical models of general host-parasite coevolution (Gandon et al. 2008) predict that if parasite evolution closely tracks host evolution, parasitic eggs should be a better match (i.e., more "infective") to host eggs from the same time period than to host eggs from a different time period. We further predicted that this difference should be most pronounced when comparing historical host eggs to current-day parasites that they have not yet encountered. Precisely such a pattern was found for egg color, as would be expected if the two parties had been closely coevolving over the duration of our study period. For egg patterning, the results were more complex, suggesting that parasitic mimicry of some host pattern traits may be improving over time, whereas in others it appears to be deteriorating relative to the host. This is plausible given that as mimicry of host eggs by parasites increases, there is no reason to expect that host discrimination and parasitic mimicry should improve (or deteriorate) for all attributes of egg appearance at the same rate. On the contrary, in the common cuckoo different features of host egg patterning (dispersion, marking size, contrast, and so on) are matched to different degrees by the various host races, which are presumably at different stages in the arms race and differ in their degree of discrimination; mimicry is effective for some attributes, yet poor for others (Stoddard and Stevens 2010).

Finally, we found that within both time periods hosts produced more extreme phenotypes than did parasites. This may result from a time lag between host and parasite adaptation, which should also prevent the system from settling to a stable polymorphism (May and McLean 2007; Schmid-Hempel 2011). A greater variance in host than in parasitic phenotypes is also one of the theoretical preconditions for coevolutionary oscillations in egg appearance to arise (Takasu 2005); the other is autosomal inheritance of egg traits, which we return to below. An alternative explanation for the finding that parasitic phenotypes were less variable than hosts' is that a degree of generalism is advantageous to parasites because it allows them to match a greater range of host phenotypes. This might be achieved under a balance of circumstances depending on the distribution of host phenotypes and the risk of rejection in relation to phenotypic difference. Our previous work has shown that although prinias are highly discriminating, they do not show the same level of refinement in rejection behavior in response to small phenotypic differences as do other, less polymorphic host species of the cuckoo finch (Spottiswoode and Stevens 2011). This may allow some level of generalization in the parasitic egg colors.

The phenotypic changes detected in this study have been observable over a period of less than 40 years, adding to the increasing number of studies detecting evolutionary change in the wild on a timescale of decades (Thompson 1998), although rapid reciprocal changes in coevolving species remain rare (Lively and Dybdahl 2000; Decaestecker et al. 2007). Yet the cuckoo finch may be an ancient species, since its lineage split from its sister group, the parasitic Vidua finches, about 20 million years ago (Sorenson and Payne 2001); hence, it may have been interacting with its prinia host over a considerable period of evolutionary time. Extrapolating the observed changes in, for example, color over even relatively short evolutionary timescales would produce extreme shifts that would quickly reach the limits of bird color space and perhaps also become disadvantageous for other reasons, such as thermoregulation or conspicuousness to predators (e.g., Lahti 2008). It is therefore surprising that such a level of phenotypic change has been detectable during the timescale of this study. These rapid changes may result from oscillations through time with respect to their distribution in phenotypic space (Takasu 2005), with phenotypes periodically returning to the same point rather than continuously growing more extreme. In our system, such oscillations might equate to changes in any given egg trait "rippling" outward in phenotypic space, 
until parasitic eggs reach sufficiently extreme phenotypes that more average host egg phenotypes become newly advantageous (contrary to the stage we currently observe for most traits). We found a "hole" in the spread of egg colors that corresponded closely to the center of the color distribution, indicating an absence of average-colored eggs. If, as we have speculated, this hole is the outcome of eggs moving outward in color space, in this framework the dynamics would equate to the hole eventually growing large enough to make average-colored eggs an evolutionary attraction for hosts because parasites have followed hosts out toward the periphery of the distribution. Any host that fell back toward the center of the distribution would then be able to increase its chances of detecting a parasite because mimetic parasites in this part of the distribution would be rare. Thus, the system may continuously oscillate within the physiological or other limits of phenotypic space, with selection alternately favoring more and less extreme egg traits. We suggest that this system is therefore more likely to follow a coevolutionary model of "fluctuating selection dynamics," involving oscillations of the frequencies of traits in each party over time owing to frequency-dependent selection (Woolhouse et al. 2002; Gandon et al. 2008), rather than the more conventional consideration that brood parasitic systems follow an "arms race dynamics" model that lacks oscillations and frequency-dependent selection and each party instead gains accumulated "improvements" over time. This would be a fruitful area of future modeling.

Given that our evidence is correlative in nature, care is needed to investigate whether egg phenotypes could in any way have been affected by unmeasured confounding factors. These include environmental effects on laying females in the wild, collection bias in the historical collection, and fading of eggs during storage of the historical collection. Environmental effects experienced by the host female are unlikely, because host and parasite have responded in different ways and because to date the few such effects on egg color that have been documented (e.g., Avilés et al. 2007) are highly subtle compared with the well-established genetic component of egg appearance (e.g., Collias 1993) and the substantial changes we have found here. Collection bias in the historical collection is also highly unlikely to account for the observed changes since the collector reported collecting all clutches. Moreover, if any bias did nonetheless occur, it should have favored unusual or extreme eggs in the historical sample (opposite to the overall pattern predicted and observed), thus rendering our results conservative. Egg fading is a potential confounding factor that needs careful consideration, but several lines of evidence suggest that it is very unlikely to account for the observed changes. First, historical eggs were collected relatively recently, stored since collection in a lightproof cab- inet, and only rarely removed for inspection in a darkened room. Second, a recent study of egg fading found that the only wavelengths affected by egg age were in the bluegreen chroma (Cassey et al. 2010; see also Moreno et al. 2011), whereas we found significant temporal changes in all four cone catches (hosts: $\left|t^{\prime}\right|>3.15, P<.002$; parasites: $\left|t^{\prime}\right|>5.16, P<.001$ ), not just the shortwave cone catch that corresponds to blue-green chroma. More work is clearly needed on the nature of egg fading in collections, including how it may be influenced by storage conditions and whether and to what degree any fading that does occur is perceptually salient to a bird, as measurements of egg fading have rarely considered avian vision. Finally, even if egg fading had affected the historical samples at all wavelengths, the observed changes in color type (e.g., proliferation of olive-green host eggs and change from red to blue parasitic eggs) cannot be explained by egg fading, nor can most changes in eggshell pattern. Our pattern estimates describe the shape of overlaid darker markings, which should be unaffected by the underlying color. An exception may be pattern contrast ("total power"), which could potentially increase in value were the background color to have faded. We therefore suggest caution in interpreting temporal change in this particular pattern trait.

Could any other source of selection have driven the observed changes? Nest predation is another major source of selection on egg appearance (reviewed by Stoddard et al. 2011), but for the following reasons it seems highly unlikely to account for our results. First, there is little reason to expect predation pressure to favor a diversification in conspicuous egg colors. While apostatic selection by predators owing to search image formation can lead to polymorphisms in prey coloration (Bond and Kamil 1998, 2002, 2006), different prey morphs should still have equal crypsis against the background in absolute terms, and selection should strongly favor a reduction in conspicuousness (Stevens and Merilaita 2011). That is clearly not the case here, given the conspicuous egg colors involved. Second, during our field study the majority of prinia egg predation events showed evidence suggestive of snake predation (no physical damage to nest, $n=117$; regurgitated eggshells alongside nest, $n=4$ ). Snakes primarily rely on olfaction and infrared to locate eggs rather than the birdvisible spectrum analyzed here. By contrast, we have little evidence that visually guided mammalian predators represent a major threat ( $n=6$ cases of physical damage to the nest). Third, prinia nests are oval in shape, such that eggs are concealed when viewed from most angles. The nest structure itself is therefore likely to be the cue used by visually guided predators, and there is no reason to expect nest structure to covary with egg appearance.

In summary, all the foregoing evidence strongly suggests that major confounding factors are unlikely to account for 
the suite of changes reported here. Nonetheless, our findings remain correlative, and in order to fully exclude the above concerns it would be ideal to study an unparasitized control population of hosts at a different location. However, time-series data over several decades of any type from natural populations are rare, and to our knowledge no such data exist for this host-parasite system, which remains little known beyond the present study site. It is difficult, moreover, to establish whether a population is genuinely unparasitized, since hosts may be proficient rejectors, leading to a lack of recorded parasitism attempts despite an evolutionary history of parasitism.

We cannot conclusively distinguish whether new phenotypes arose from selection or gene flow. At present we have no knowledge of any geographical structure in the cuckoo finch-host system that could generate wider-scale spatial variation in phenotypes, but we note that population densities at our study site are high, which should not favor net gene flow into the population (Lenormand 2002). Regardless, it is difficult to explain our results on the basis of gene flow alone, since gene flow should counter local adaptation (Lenormand 2002) and we have found clear support for our predictions on the basis of adaptive host-parasite interactions. In particular, the results of the time-shift analyses would be very hard to explain in the absence of selection, as would the magnitude and consistency of the observed changes. The genetic mechanisms underlying egg polymorphisms in both species also remain unknown, but autosomal inheritance of egg coloration was found in the only bird species with polymorphic eggs studied to date (Collias 1993), and we might further speculate that the autosomal genes involved should be unlinked in the present system. Autosomal inheritance of both egg color and pattern would, via recombination, also allow for (i) more rapid evolutionary change, again in a close analogy of Red Queen dynamics, and (ii) the observed lack of phenotypic correlation between different aspects of egg appearance. Better understanding of the mode of inheritance of egg traits would greatly inform modeling of the evolutionary dynamics of the prinia-cuckoo finch arms race.

By using models of avian vision and digital image analysis, we have been able to detect changes that have occurred on multiple and independent features of egg phenotype. This supports other recent studies that have demonstrated how linking sensory biology, ecology, and behavior can enhance our knowledge of evolutionary processes (e.g., Seehausen et al. 2008). Overall, the priniacuckoo finch arms race strongly suggests that frequencydependent selection through brood parasitism can be a potent force in producing and maintaining extreme levels of phenotypic polymorphism in nature.

\section{Acknowledgments}

We dedicate this article to the memory of Major John Colebrook-Robjent (1935-2008). In Zambia, we thank the Zambia Wildlife Authority for permits; the Bruce-Miller, Counsell, Danckwerts, Duckett, and Robjent families for their hospitality; and L. Hamusikili, K. Mono, C. Moya, A. Munkombwe, C. Munkombwe, R. Munkombwe, S. Munkombwe, M. Siababwa, and D. Siyapolo for finding nests. For helpful discussion and comments, we thank T. Birkhead, M. Broom, N. Davies, R. Kilner, G. Ruxton, M. C. Stoddard, W. Sutherland, and three anonymous reviewers. C.N.S. was funded by a Royal Society Dorothy Hodgkin Research Fellowship, Sidney Sussex College and Newnham College, Cambridge, United Kingdom, and the Department of Science and Technology/National Research Foundation Centre of Excellence at the Percy FitzPatrick Institute, University of Cape Town, Rondebosch, South Africa. M.S. was funded by a Biotechnology and Biological Sciences Research Council David Phillips Research Fellowship (BB/G022887/1).

\section{Literature Cited}

Allen, J. A. 1988. Frequency dependent selection by predators. Philosophical Transactions of the Royal Society B: Biological Sciences 319:485-503.

Allen, J. A., and B. Clarke. 1968. Evidence for apostatic selection by wild passerines. Nature 220:501-502.

Apanius, V., D. Penn, P. R. Slev, L. R. Ruff, and W. K. Potts. 1997. The nature of selection on the major histocompatibility complex. Critical Reviews in Immunology 17:179-224.

Avilés, J. M. 2008. Egg colour mimicry in the common cuckoo Cuculus canorus as revealed by modelling host retinal function. Proceedings of the Royal Society B: Biological Sciences 275:23452352.

Avilés, J. M., B. G. Stokke, A. Moksnes, E. Røskaft, and A. P. Møller. 2007. Environmental conditions influence egg color of reed warblers Acrocephalus scirpaceus and their parasite, the common cuckoo Cuculus canorus. Behavioral Ecology and Sociobiology 61: 475-485.

Beecher, M. D. 1982. Signature systems and kin recognition. American Zoology 22:477-490.

Bond, A. B., and A. C. Kamil. 1998. Apostatic selection by blue jays produces balanced polymorphism in virtual prey. Nature 395:594596.

2002. Visual predators select for crypticity and polymorphism in virtual prey. Nature 415:609-613.

-2006. Spatial heterogeneity, predator cognition, and the evolution of color polymorphism in virtual prey. Proceedings of the National Academy of Sciences of the USA 103:3214-3219.

Brooke, M. d. L., and N. B. Davies. 1988. Egg mimicry by cuckoos Cuculus canorus in relation to discrimination by hosts. Nature 335: 630-632.

Cassey, P., M. Honza, T. Grim, and M. E. Hauber. 2008. The modelling of avian visual perception predicts behavioural rejection responses to foreign egg colours. Biology Letters 4:515-517.

Cassey, P., G. Maurer, C. Duval, J. G. Ewen, and M. E. Hauber. 2010. 
Impact of time since collection on avian eggshell color: a comparison of museum and fresh egg specimens. Behavioral Ecology and Sociobiology 64:1711-1720.

Collias, E. C. 1993. Inheritance of egg-colour polymorphisms in the village weaver (Ploceus cucullatus). Auk 110:683-692.

Cuthill, I. C. 2006. Color perception. Pages 3-40 in G. E. Hill and K. J. McGraw, eds. Bird coloration. Vol. 1. Mechanisms and measurement. Harvard University Press, Cambridge, MA.

Dale, J., D. B. Lank, and H. K. Reeve. 2001. Signaling individual identity versus quality: a model and case studies with ruffs, queleas, and house finches. American Naturalist 158:75-86.

Davies, N. B., and M. D. Brooke. 1989. An experimental study of co-evolution between the cuckoo, Cuculus canorus, and its hosts. II. Host egg markings, chick discrimination and general discussion. Journal of Animal Ecology 58:225-236.

Dawkins, R., and J. R. Krebs. 1979. Arms races between and within species. Proceedings of the Royal Society B: Biological Sciences 205:489-511.

Decaestecker, E., S. Gaba, J. Raeymaekers, R. Stoks, L. Van Kerckhoven, D. Ebert, and L. De Meester. 2007. Host-parasite "Red Queen" dynamics archived in pond sediment. Nature 450:870 873.

Dieckmann, U., P. Marrow, and R. Law. 1995. Evolutionary cycling in predator-prey interactions: population dynamics and the Red Queen. Journal of Theoretical Biology 176:91-102.

Ehrlich, P. R., and P. H. Raven. 1964. Butterflies and plants: a study in coevolution. Evolution 18:586-608.

Endler, J. A. 1986. Natural selection in the wild. Princeton University Press, Princeton, NJ.

Endler, J. A., and P. W. J. Mielke. 2005. Comparing color patterns as birds see them. Biological Journal of the Linnean Society 86: 405-431.

Gaba, S., and D. Ebert. 2009. Time-shift experiments as a tool to study antagonistic coevolution. Trends in Ecology \& Evolution 24: 226-232.

Gandon, S., A. Buckling, E. Decaestecker, and T. Day. 2008. Hostparasite coevolution and patterns of adaptation across time and space. Journal of Evolutionary Biology 21:1861-1866.

Gorchein, A., C. K. Lima, and P. Cassey. 2009. Extraction and analysis of colourful eggshell pigments using HPLC and HPLC/electrospray ionization tandem mass spectrometry. Biomedical Chromatography 23:602-606.

Hanlon, R. T., C.-C. Chiao, L. M. Mäthger, A. Barbosa, K. C. Buresch, and C. Chubb. 2009. Cephalopod dynamic camouflage: bridging the continuum between background matching and disruptive coloration. Philosophical Transactions of the Royal Society B: Biological Sciences 364:429-437.

Hart, N. S., J. C. Partridge, A. T. D. Bennett, and I. C. Cuthill. $2000 a$. Visual pigments, cone oil droplets and ocular media in four species of estrildid finch. Journal of Comparative Physiology A 186:681694.

Hart, N. S., J. C. Partridge, I. C. Cuthill, and A. T. D. Bennett. 2000 b. Visual pigments, oil droplets, ocular media and cone photoreceptor distribution in two species of passerine: the blue tit (Parus caeruleus L.) and the blackbird (Turdus merula L.). Journal of Comparative Physiology A 186:375-387.

Kennedy, G. Y., and H. G. Vevers. 1976. A survey of avian eggshell pigments. Comparative Biochemistry and Physiology B 55:117123.

Komdeur, J., M. Oorebeek, T. van Overveld, and I. C. Cuthill. 2005. Mutual ornamentation, age, and reproductive performance in the European starling. Behavioral Ecology 16:805-817.
Lahti, D. C. 2005. Evolution of bird eggs in the absence of cuckoo parasitism. Proceedings of the National Academy of Sciences of the USA 102:18057-18062.

. 2006. Persistence of egg recognition in the absence of cuckoo brood parasitism: pattern and mechanism. Evolution 60:157-168.

- 2008. Population differentiation and rapid evolution of egg color in accordance with solar radiation. Auk 125:796-802.

Lenormand, T. 2002. Gene flow and the limits to natural selection. Trends in Ecology \& Evolution 17:183-189.

Lively, C. M., and M. F. Dybdahl. 2000. Parasite adaptation to locally common host genotypes. Nature 405:679-681.

Lund, U., and C. Agostinelli. 2007. Circular: circular statistics. R package version $0.3-8$.

Martin, S. J., H. Helanterä, and F. P. Drijfhout. 2011. Is parasite pressure a driver of chemical cue diversity in ants? Proceedings of the Royal Society B: Biological Sciences 278:496-503.

May, R., and A. McLean. 2007. Theoretical ecology: principles and applications. Oxford University Press, Oxford.

Moksnes, A., E. Røskaft, A. T. Braa, L. Korsnes, H. L. Lampe, and H. C. Pedersen. 1990. Behavioural responses of potential hosts towards artificial cuckoo eggs and dummies. Behaviour 116:6489.

Moreno, J., E. Lobato, and J. Morales. 2011. Eggshell blue-green colouration fades immediately after oviposition: a cautionary note about measuring natural egg colours. Ornis Fennica 88:51-56.

Newton, A. 1896. A dictionary of birds. Adam \& Charles Black, London.

Nuismer, S. L., M. Doebeli, and D. Browning. 2005. The coevolutionary dynamics of antagonistic interactions mediated by quantitative traits with evolving variances. Evolution 59:2073-2082.

Ödeen, A., and O. Håstad. 2003. Complex distribution of avian color vision systems revealed by sequencing the SWS1 opsin from total DNA. Molecular Biology and Evolution 20:855-861.

Olendorf, R., F. H. Rodd, D. Punzalan, A. E. Houde, C. Hurt, D. N. Reznick, and K. A. Hughes. 2006. Frequency-dependent survival in natural guppy populations. Nature 441:633-636.

Osorio, D., and M. Vorobyev. 2005. Photoreceptor spectral sensitivities in terrestrial animals: adaptations for luminance and colour vision. Proceedings of the Royal Society B: Biological Sciences 272: $1745-1752$.

Pietrewicz, A. T., and A. C. Kamil. 1979. Search image formation in the blue jay (Cyanocitta cristata). Science 204:1332-1333.

R Development Core Team. 2010. R: a language and environment for statistical computing. R Foundation for Statistical Computing, Vienna.

Ruxton, G. D. 2006. The unequal variance $t$-test is an underused alternative to Student's $t$-test and the Mann-Whitney $U$ test. Behavioral Ecology 17:688-690.

Schmid-Hempel, P. 2011. Evolutionary parasitology. Oxford University Press, Oxford.

Seehausen, O., Y. Terai, I. S. Magalhaes, K. L. Carleton, H. D. J. Mrosso, R. Miyagi, I. Van der Sluijs, et al. 2008. Speciation through sensory drive in cichlid fish. Nature 455:620-627.

Siddiqi, A., T. W. Cronin, E. R. Loew, M. Vorobyev, and K. Summers. 2004. Interspecific and intraspecific views of color signals in the strawberry poison frog Dendrobates pumilio. Journal of Experimental Biology 207:2471-2485.

Sorenson, M. D., and R. B. Payne. 2001. A single, ancient origin of obligate brood parasitism in African finches: implications for hostparasite coevolution. Evolution 55:2550-2567.

Spottiswoode, C. N., and M. Stevens. 2010. Visual modeling shows that avian host parents use multiple visual cues in rejecting par- 
asitic eggs. Proceedings of the National Academy of Sciences of the USA 107:8672-8676.

2011. How to evade a coevolving brood parasite: egg discrimination versus egg variability as host defences. Proceedings of the Royal Society B: Biological Sciences 278:3566-3573.

Stevens, M., and S. Merilaita, eds. 2011. Animal camouflage: from mechanisms to function. Cambridge University Press, Cambridge.

Stevens, M., A. Párraga, I. C. Cuthill, J. C. Partridge, and T. Troscianko. 2007. Using digital photography to study animal coloration. Biological Journal of the Linnean Society 90:211-237.

Stoddard, M. C., and R. O. Prum. 2008. Evolution of avian plumage color in a tetrahedral colour space: a phylogenetic analysis of $\mathrm{New}$ World buntings. American Naturalist 171:755-776.

Stoddard, M. C., and M. Stevens. 2010. Pattern mimicry of host eggs by the common cuckoo, as seen through a bird's eye. Proceedings of the Royal Society B: Biological Sciences 277:1387-1393.

. 2011. Avian vision and the evolution of egg color mimicry in the common cuckoo. Evolution 65:2004-2013.

Stoddard, M. C., K. Marshall, and R. M. Kilner. 2011. Imperfectly camouflaged eggs: artefact or adaptation? Avian Biology Research 4:196-213.

Stokke, B. G., A. Moksnes, and E. Røskaft. 2002. Obligate brood parasites as selective agents for the evolution of egg appearance in passerine birds. Evolution 56:199-205.

Summers, K., S. McKeon, J. Sellars, M. Keusenkothen, J. Morris, D. Gloeckner, C. Pressley, et al. 2003. Parasitic exploitation as an engine of diversity. Biological Review 78:639-675.

Swynnerton, C. F. M. 1918. Rejections by birds of eggs unlike their own: with remarks on some of the cuckoo problems. Ibis 6:127154.

Takasu, F. 2003. Co-evolutionary dynamics of egg appearance in avian brood parasitism. Evolutionary Ecology Research 5:345-362.
2005. A theoretical consideration on co-evolutionary interactions between avian brood parasites and their hosts. Ornithological Science 4:65-67.

Thompson, J. N. 1998. Rapid evolution as an ecological process. Trends in Ecology \& Evolution 18:329-332.

. 1999. The evolution of species interactions. Science 284: 2116-2118.

Van Valen, L. 1973. A new evolutionary law. Evolutionary Theory 1: $1-30$.

Vernon, C. J. 1964. The breeding of the cuckoo weaver Anomalospiza imberbis in southern Rhodesia. Ostrich 35:260-263.

Victoria, J. K. 1972. Clutch characteristics and egg discriminative ability of the African village weaverbird Ploceus cucullatus. Ibis 114: 367-376.

Vorobyev, M., and D. Osorio. 1998. Receptor noise as a determinant of colour thresholds. Proceedings of the Royal Society B: Biological Sciences 265:351-358.

Woolhouse, M. E. J., J. P. Webster, E. Domingo, B. Charlesworth, and B. R. Levin. 2002. Biological and biomedical implications of the co-evolution of pathogens and their hosts. Nature Genetics 32:569-577.

Yang, C., W. Liang, Y. Cai, S. Shi, F. Takasu, A. P. Møller, A. Antonov, et al. 2010. Coevolution in action: disruptive selection on egg colour in an avian brood parasite and its host. PLoS ONE 5:e10816.

Yoder, J. B., and S. L. Nuismer. 2010. When does coevolution promote diversification? American Naturalist 176:802-817.

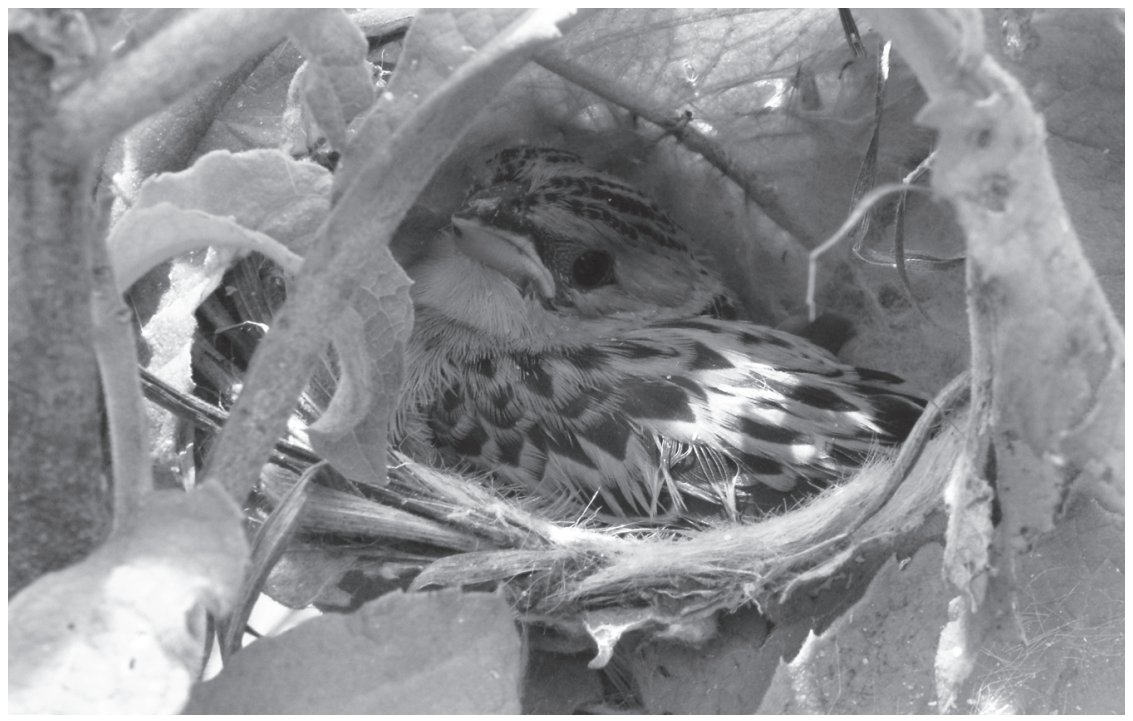

Center, Cuckoo finch chick monopolizing a host nest, which happens if hosts fail to spot a parasite egg. This cuckoo finch chick is nearly fully grown and is about to fledge from a red-faced cisticola nest. Photograph by Claire Spottiswoode. A color version of this figure is available in the online edition of the American Naturalist. 